\section{Inhibition thérapeutique de mTOR dans la polykystose rénale autosomique dominante}

Dominique Joly
Université René Descartes - Paris V, Service de néphrologie, Hôpital Necker-Enfants malades, 149, rue de Sèvres, 75015 Paris, France. doume.joly@gmail.com
$>$ Avec une incidence dans la population générale proche de $1 / 1000$, la polykystose rénale autosomique dominante (PKRAD) est l'une des maladies héréditaires monogéniques humaines les plus fréquentes. Elle se révèle habituellement à l'âge adulte et est principalement caractérisée par l'apparition et le développement progressif de multiples kystes rénaux qui augmentent le volume rénal total et sont potentiellement source de complications: douleur, hématurie, lithiase, infection urinaire, hypertension artérielle et surtout développement d'une insuffisance rénale qui parvient au stade terminal chez $50 \%$ des individus atteints après l'âge de 60 ans.

\section{Conséquences des mutations des gènes PKD1 et PKD2}

Les mutations des gènes $P K D 1$ et $P K D 2$ sont responsables de respectivement $85 \%$ et $15 \%$ des cas de PKRAD. Ces gènes codent respectivement les polycystines 1 et 2. Ces deux protéines sont principalement localisées dans le cil primaire non motile des cellules tubulaires rénales où elles interagissent, coopèrent et pourraient transformer par mécanotransduction la perception du flux urinaire en une signalisation, dominée par un afflux calcique intracellulaire [1].

Au cours des dernières années, la compréhension des mécanismes moléculaires associés à la kystogenèse a permis de définir plusieurs stratégies thérapeutiques pour cette maladie (Figure 1). Une stratégie directe consiste à rétablir le signal calcique provenant des cils primaires grâce à des agonistes des poly- cystines. Le triptolide ${ }^{1}$ rétablit dans les cellules tubulaires déficientes en polycystine $1\left(\mathrm{Pkdl}^{-/-}\right)$une entrée de calcium par un mécanisme dépendant de la polycystine 2 et bloque la croissance habituellement excessive de ces cellules. Dans un modèle murin de PKRAD présentant un phénotype kystique dans les premiers jours de vie ( $P k d l^{f l o x /-}$; Ksp-Cre $)$, ce produit réduit efficacement la kystogenèse. Un essai randomisé est actuellement en cours chez l'homme [2].

Les stratégies indirectes consistent à agir, en aval des polycystines, sur des voies de signalisation dérégulées dans le PKRAD. Ainsi des interventions sur les voies de I'AMP cyclique (AMPC) et de mTOR (mammalian tar get of rapamycin) ont donné lieu à plusieurs essais thérapeutiques, en cours ou récemment publiés.

Les fortes concentrations d'AMPc intracellulaire observées dans les cellules tubulaires rénales kystiques stimuleraient la kystogenèse via une augmentation de la prolifération cellulaire et/ ou une augmentation de la sécrétion chlorée intrakystique. Deux types de traitements peuvent réduire la synthèse d'AMPc par les cellules tubulaires rénales : les antagonistes des récepteurs V2R à la vasopressine (tolvaptan) et les analogues de la somatostatine (octréotide, lanréotide) [3, 4]. L'efficacité de ces produits est actuellement testée contre placebo sur l'évolution à 3 ans du volume rénal total mesuré par IRM (imagerie par résonance magnétique).

${ }^{1}$ Le triptolide est dérivé d'une herbe chinoise, Tripterygium wilfordii.
En 2006, Shillingford et al. ont montré que mTOR, une sérine-thréonine kinase qui régule la croissance et la prolifération cellulaire, était anormalement activée dans les cellules épithéliales qui bordent des kystes de patients PKRAD. Plusieurs expériences ont suggéré que la polycystine 1 était (en coopération avec la tubérine) un inhibiteur physiologique de mTOR. Les auteurs ont montré que l'utilisation d'un inhibiteur de mTOR permettait de réduire drastiquement la formation des kystes dans trois modèles animaux de PKRAD. Enfin, sachant que les inhibiteurs de mTOR (sirolimus et évérolimus) sont utilisés de longue date en tant qu'immunosuppresseurs en transplantation d'organe [9], l'analyse rétrospective de patients PKRAD ayant reçu un greffon rénal a révélé que ces produits étaient associés à une diminution de la taille des reins natifs significativement plus importante qu'avec les autres immunosuppresseurs [5]. Cette publication particulièrement riche en données a encouragé la mise en route de deux essais prospectifs randomisés humains dont les résultats viennent d'être publiés de façon conjointe dans le New England Journal of Medicine [6, 7].

\section{Résultats décevants} de l'inhibition de mTOR dans la PKRAD Dans l'étude de G. Walz et al. [6], 433 patients PKRAD ont reçu en double insu soit un placebo, soit de l'évérolimus. Sous évérolimus, l'augmentation du volume rénal total mesuré par IRM a été moins importante que sous placebo, avec des différences en valeur absolue modestes (augmentation de 


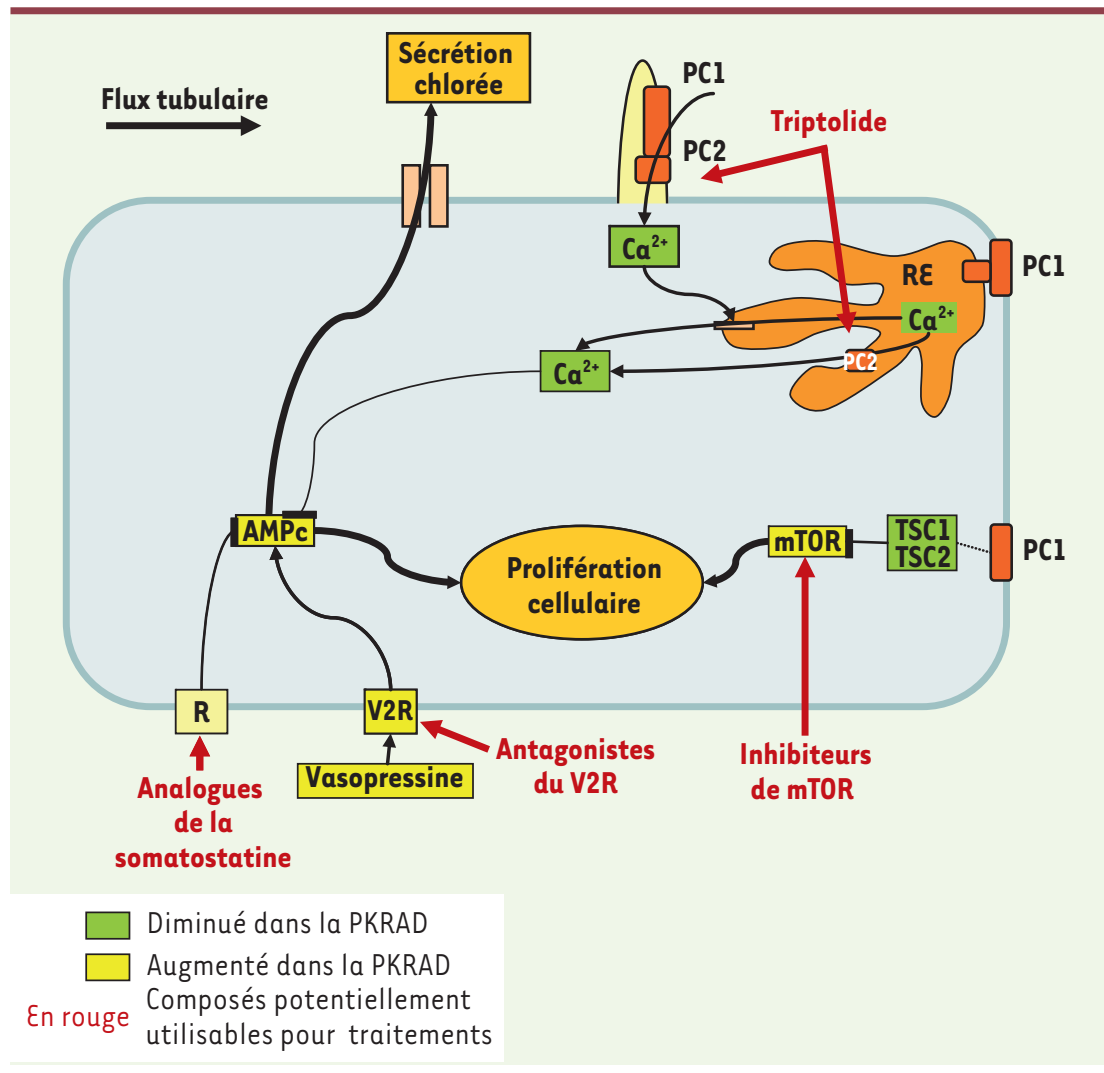

$102 \mathrm{ml}$ versus $157 \mathrm{ml}$ à un an, $p=0,02$; et de $230 \mathrm{ml}$ versus $301 \mathrm{ml}$ à deux ans, $p=0,06)$. Malgré cet effet sur le volume, il n'y a, de façon surprenante, pas eu d'effet sur la fonction rénale: le déclin du débit de filtration glomérulaire (DFG) à 24 mois était comparable dans les deux groupes (déclin de $8,9 \mathrm{ml}$ par minute pour une surface corporelle de $1,73 \mathrm{~m}^{2}$ versus $7,7 \mathrm{ml}$ par minute $/ 1,73 \mathrm{~m}^{2}$, $p=0,15)$. Dans l'étude de Serra et al. [7], 100 patients PKRAD ont reçu $2 \mathrm{mg}$ de sirolimus ou des soins conventionnels. Les patients inclus dans cette étude étaient plus jeunes que dans l'étude de Walz (10 ans de moins en moyenne) et avaient une maladie rénale moins avancée (DFG > $70 \mathrm{ml} /$ minute $/ 1,73 \mathrm{~m}^{2}$ ). Après 18 mois, le sirolimus n'a significativement modifié ni le volume rénal ni la fonction rénale.

Deux enseignements majeurs doivent être tirés des résultats décevants de l'inhibition de mTOR dans la PKRAD. Le premier enseignement est que la posologie utilisée chez l'homme était probablement insuffisante. G. Canaud et al. ont en effet rapporté récemment une observation exceptionnelle chez un homme ayant reçu un greffon rénal provenant d'un individu jeune porteur d'une PKRAD débutante. Le sirolimus (utilisé en tant qu'agent immunosuppresseur, avec des taux sériques conventionnels compris entre 3 et $8 \mathrm{ng} / \mathrm{ml}$ ) a permis de bloquer l'activation de mTOR dans les cellules sanguines mononucléées, mais pas dans les cellules épithéliales formant les kystes rénaux qui conservaient une forte activité mTOR [8]. Les posologies utilisées dans les études animales étaient en comparaison nettement plus élevées. De telles posologies ne pourront hélas pas être atteintes chez l'homme de façon réaliste.

Le deuxième enseignement est que la volumétrie rénale n'est pas un bon critère d'efficacité des traitements. L'étude de Walz et al. [6] a montré clairement que, contrairement à ce que l'on a longtemps pensé de façon simpliste: (1) il n'y a pas de corrélation solide entre le volume rénal et la fonction rénale ; (2) une progression du volume rénal moindre ne
Figure 1. Représentation schématique des voies de signalisation potentiellement impliquées dans la polykystose rénale autosomique dominante et ciblées par des molécules en cours d'évaluation dans le cadre d'essais thérapeutiques. Une cible directe: rétablir l'action des polycystines déficientes (avec le triptolide, agoniste de la polycystine 2); des cibles indirectes : bloquer des voies de signalisation intracellulaires surexprimées par les cellules polykystiques (AMPc, voie mTOR). PCl, polycystine 1 ; $P C 2$, polycystine 2 ; RE, réticulum endoplasmique; AMPc, AMP cyclique; V2R, récepteur $V 2$ de la vasopressine; $R$, récepteur de la somatostatine; mTOR, mammalian target of rapamycin; $\mathrm{TSCl}$ et 2, tubérine 1 et 2 .

s'accompagne pas d'un déclin diminué de la fonction rénale.

D'autres essais humains impliquant des agents thérapeutiques existants devront donc être mis en œuvre dans les années à venir. La volumétrie rénale n'étant pas un critère intermédiaire pertinent, les essais devront être prolongés et basés sur l'évolution de la fonction rénale. Parallèlement, la recherche translationnelle doit se poursuivre. II faut en effet que les cliniciens soient prêts à admettre que le déclin de la fonction rénale dans la PKRAD n'est pas nécessairement dû à un phénomène prolifératif des cellules épithéliales tubulaires et que des mécanismes lésionnels périkystiques - qui restent à découvrir - entrent en jeu. $\diamond$

Therapeutic mTOR inhibition

in autosomal dominant

polycystic kidney disease

\section{CONFLIT D'INTÉRÊTS}

L'auteur déclare avoir participé à des interventions ponctuelles pour l'entreprise Novartis.

\section{RÉFÉRENCES}

1. Torres VE, Harris PC. Autosomal dominant polycystic kidney disease: the last 3 years. Kidney Int 2009 . 76: 149-68.

2. Leuenroth SJ, Okuhara D, Shotwell JD, et al. Triptolide is a traditional Chinese medicine-derived inhibitor of polycystic kidney disease. Proc Natl Acad Sci USA 2007 ; $104:$ 4389-94. 
3. Wang X, Wu Y, Ward CJ, et al. Vasopressin directly regulates cyst growth in polycystic kidney disease. J Am Soc Nephrol 2008 ; 19 : 102-8.

4. Van Keimpema L, Nevens F, Vanslembrouck R, et al. Lanreotide reduces the volume of polycystic liver: a randomized, double-blind, placebo-controlled trial. Gastroenterology 2009; 137 : 1661-8.

5. Shillingford JM, Murcia NS, Larson $\mathrm{CH}$, et al. The mTOR pathway is regulated by polycystin- 1 , and its inhibition reverses renal cystogenesis in polycystic kidney disease. Proc Natl Acad Sci USA 2006 ; $103: 5466-71$.

6. Walz G, Budde K, Mannaa M, et al. Everolimus in patients with autosomal dominant polycystic kidney disease. N Engl J Med $2010 ; 363: 830-40$.

7. Serra AL, Poster D, Kistler AD, et al. Sirolimus and kidney growth in autosomal dominant polycystic kidney disease. $N$ Engl J Med $2010 ; 363: 820-9$.

\section{NOUVELLE}

\section{Le BDNF}

\section{Un nouvel acteur de la croissance placentaire et fotale}

Sylvain Mayeur, Marie-Amélie Lukaszewski, Christophe Breton, Laurent Storme, Claudine Junien, Didier Vieau, Jean Lesage
$>$ Chez l'homme, un poids de naissance trop faible (inférieur à 2,5 kg à terme pour un individu de type caucasien) ou trop important (supérieur à 4,5 kg) est le signe respectivement d'un retard de croissance intra-utérin (RCIU) ou d'une macrosomie fœtale. Ces altérations de croissance sont maintenant reconnues comme étant des facteurs de prédisposition à l'apparition de pathologies métaboliques à l'âge adulte telles que l'obésité, le diabète de type 2, l'hypertension artérielle ou encore certaines maladies neurologiques. La croissance fœtale est majoritairement dépendante du placenta qui conditionne l'environnement intrautérin car il fournit au fœtus des nutriments et de l'oxygène mais élimine aussi de sa circulation des composés toxiques issus de son métabolisme [1]. De nombreuses données ont mis en évidence certains dommages morphofonctionnels du placenta lors d'altérations de la croissance fœtale, mais des données plus récentes indiquent que celle-ci pourrait aussi impliquer d'autres facteurs comme des adipocytokines ou encore, de manière plus surprenante, la neurotrophine BDNF (brain-derived neurotrophic factor).

\section{Biologie du BDNF}

Le BDNF est un membre de la famille des neurotrophines qui comprend également le NGF (nerve growth factor) et les neurotrophines 3 et 4/5. L'action du BDNF est relayée par deux types de récepteurs: TrkB, un récepteur à activité tyrosine kinase de haute affinité et $\mathrm{p} 75$, de plus

$(\rightarrow)$ Voir $\mathrm{m} / \mathrm{s} \mathrm{n}^{\circ} 2$, faible affinité $(\rightarrow)$ [11].

Durant la période périnatale, le BDNF contrôle le développement et la maturation du système nerveux central (SNC). À l'âge adulte, il est plutôt impliqué dans les processus de plasticité neuronale et synaptique mais aussi dans la neurotransmission [2]. Bien que principalement exprimé dans le SNC, le BDNF joue également un rôle dans d'autres tissus en périphérie. Par exemple, il semble impliqué dans la régulation de l'homéostasie glucidique, mais aussi dans les processus liés à l'angiogenèse [3]. La découverte de l'expression du système BDNF/TrkB dans le placenta [4] suggérait son implication dans le développement placentaire et fœtal, ce qui a, de fait, été démontré récemment.

\section{BDNF et gestation}

Chez le rat et la souris, le BDNF est exprimé dans le trophospongium du placenta, qui représente une zone tissulaire d'origine maternelle. Le récepteur TrkB est, quant à lui, exprimé dans le trophospongium mais aussi dans la zone
8 Canaud G, Knebelmann B, Harris PC, et al. Therapeutic mTOR inhibition in autosomal dominant polycystic kidney disease: what is the appropriate serum level? Am J Transplant $2010 ; 10$ : 1701-6.

9. Pallet $N$, Beaune $P$, Thervet $\varepsilon$, et al. Inhibiteurs de mTOR : des antiprolifératifs pléiotropiques. Med Sci (Paris) $2006 ; 22:$ 947-52.

S. Mayeur, M.A. Lukaszewski, C. Breton, L. Storme, D. Vieau, J. Lesage : Unité environnement périnatal et croissance, EA4489, USTL, Universités de Lille 1 et Lille 2, bâtiment SN4,

59655 Villeneuve d'Ascq, France.

C. Junien : Biologie du développement et reproduction, UMR INRA-ENVA-CNRS, 78352 Jouy en Josas, France. jean.lesage@univ-lillel.fr

labyrinthique qui constitue la principale zone d'échange vasculaire fœto-maternelle $[4,5]$. Une étude combinant des expérimentations menées in vitro et in vivo chez des souris vient de démontrer l'importance du BDNF dans le développement placentaire et fœtal. Ainsi, le traitement de cellules trophoblastiques placentaires par un inhibiteur pharmacologique des récepteurs Trk (le k252a) ou encore par l'ectodomaine TrkB, diminue la prolifération des trophoblastes et augmente l'apoptose. Ces résultats ont été confirmés in vivo chez des souris gestantes et ont clairement démontré que I'inhibition du système BDNF/TrkB diminuait l'épaisseur de la zone labyrinthique placentaire, ce qui a pour conséquence de diminuer la croissance des fœtus [4]. Chez le rat, dans des modèles de RCIU induit par une restriction calorique ou protéique maternelle, il a été montré que le retard de croissance fœtale est associé à une diminution de l'expression du BDNF placentaire et à une augmentation de l'expression de son récepteur $\operatorname{TrkB}[5,6]$.

Chez l'homme, l'expression placentaire du couple BDNF/TrkB n'est pas modulée au cours de la grossesse non pathologique [7]. Cependant, elle est augmentée lorsque la grossesse est associée à un retard de croissance fœtale intra- 